\title{
A Parameterization of the Chandra Point Spread Function
}

\author{
Christopher L. Allen, Diab Jerius, and Terry Gaetz \\ Smithsonian Astrophysical Observatory, 60 Garden St., Cambridge, MA 02138
}

\begin{abstract}
The Chandra X-ray Observatory point spread function (PSF) is a complex function of source position and energy. On-orbit calibration observations with sufficient $\mathrm{S} / \mathrm{N}$ sample only a small fraction of the possible parameter space, and are complicated by detector systematics. Thus, the standard method of analyzing Chandra data uses the standard Chandra optics model as a reference. The optics model accurately simulates the telescope's PSF, but as it is a raytrace based technique, it can be time-consuming to run and is not always appropriate for a given analysis task. A simple parameterization of the PSF would be useful for many analysis purposes, in many cases obviating the need for users to run lengthy raytraces. We present an approach to a simple PSF parameterization of off-axis point sources, discussing its applicability to analysis of Chandra observations in light of the complicated PSF structure. We also present some results of our PSF parameterization and discuss its accuracy.
\end{abstract}

Keywords: Chandra, HRMA, PSF, encircled energy function, ellipses, moments

\section{INTRODUCTION}

Previous parameterizations of the Chandra PSF have used the encircled energy function, characterizing the PSF with the fractional energy measured within a circular aperture as a function of radius. Chandra's PSF in reality is not circular, it becomes elongated with increasing off-axis angle. Elliptical apertures would thus be better representatives of the underlying PSF. We have developed a technique of deriving a series of elliptical regions that have been fit to the PSF, and comparing these to the total number of counts incident on the detector in the raytrace. This enclosed count fraction (ECF) is analogous to the encircled energy. The ECF and the properties of the elliptical regions from which it was derived provide a parameterization of the Chandra PSF that can be compared to real observations. The determined ellipses are representative of the large scale PSF. However, our method produces nonsensical results in the PSF core, which devolves into multiple components at sufficiently high off-axis source positions. Figure 1 illustrates the complexity of the off-axis core,

When completed, the parameterization of the Chandra PSF described in this paper will be presented to users as a standard calibration product.

\section{SIMULATIONS}

A set of simulated point source PSFs were created with the SAOsac ${ }^{1}$ raytrace simulation software, having a range of monochromatic energies and a set of positions covering a polar grid of pointings. For each simulation, the rays were passed through the Chandra optics (the High Resolution Mirror Assembly, ${ }^{2}$ or HRMA) and projected onto the detector plane but were not pixelized. Ghost rays were suppressed in order to increase the accuracy of the fitted ellipses. In the current study we concentrate on the HRC-I detector ${ }^{3}$ to simplify the analysis (the HRC-I is the simplest detector on Chandra). The HRC-I model includes detector geometry, energy response, and pixel resolution, and simulates an aspect solution blur by adding a simple Gaussian blur to the

Send correspondence to DJ; E-mail: djerius@cfa.harvard.edu Copyright 2004 Society of Photo-Optical Instrumentation Engineers.

This paper was published in X-Ray and Gamma-Ray Instrumentation for Astronomy XIII, Flanagan, Kathryn A.; Siegmund, Oswald H. W., Eds, Proceedings of SPIE Vol. 5165, p. 423, and is made available as an electronic reprint with permission of SPIE. One print or electronic copy may be made for personal use only. Systematic or multiple reproduction, distribution to multiple locations via electronic or other means, duplication of any material in this paper for a fee or for commercial purposes, or modification of the content of the paper are prohibited. 

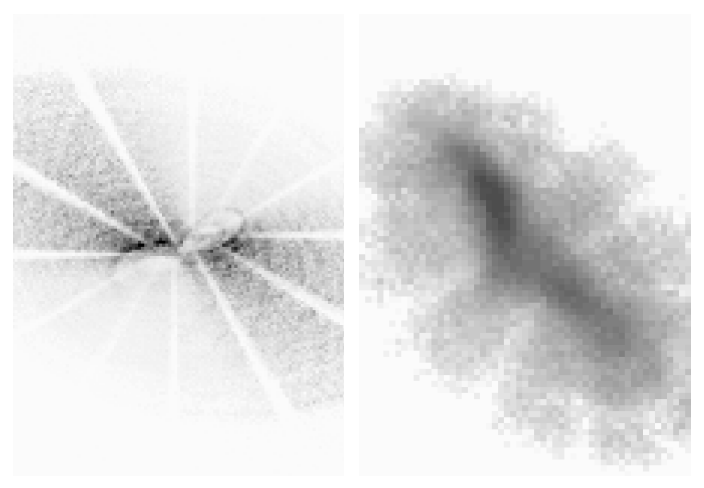

Figure 1. A selection of complex features in the Chandra PSF core. Note the two blob features in core of each image, which tend to confound our ellipse generation technique when the ellipses approach the size of the core.

rays. It also simulates the intra-pixel randomization caused by reprojecting rays from the detector plane into the sky plane by randomizing event position within the HRC-I pixel.

As a means of calibrating our results, we prepared simulations which exactly matched the pointings for a set of Chandra off-axis point source observations.

\section{ELLIPSE FITTING TECHNIQUE}

Ellipses were fit to images derived from the raytraces. The images were binned such that they had high enough $\mathrm{S} / \mathrm{N}$ to provide convergent fits, but retained as much detail as possible. The binning size ranged from slightly less than a HRC-I pixel to $2 "$, and increased with off-axis angle. Images containing $99 \%$ of the rays were created from each simulation. We preserved correct ECF values by using the total counts incident on the detector, rather than the number of rays in the $99 \%$ image as the denominator in the ECF (see below).

The $0^{\text {th }}, 1^{\text {st }}$, and $2^{\text {nd }}$ order moments are calculated from the simulated image:

$$
\mu_{i j}=\iint d x d y f(x, y) x^{i} y^{j} \Longrightarrow \sum_{x} \sum_{y} f(x, y) x^{i} y^{j}
$$

where $f(x, y)$ is the image pixel value at $(x, y)$, the integral on the left side of the arrow is the moment's analytic form, and the sum on the right side of the arrow is the moment's discrete form, which we use in analyzing our images. The parameters defining the ellipse are constructed from these calculated moments ${ }^{4}$ :

$$
\begin{gathered}
\bar{x}=\mu_{10} / \mu_{00}, \\
\bar{y}=\mu_{01} / \mu_{00}, \\
a=\left(\frac{\mu_{20}+\mu_{02}+\left[\left(\mu_{20}-\mu_{02}\right)^{2}+4 \mu_{11}^{2}\right]^{1 / 2}}{\mu_{00} / 2}\right)^{1 / 2}, \\
b=\left(\frac{\mu_{20}+\mu_{02}-\left[\left(\mu_{20}-\mu_{02}\right)^{2}+4 \mu_{11}^{2}\right]^{1 / 2}}{\mu_{00} / 2}\right)^{1 / 2}, \\
\phi=(1 / 2) \tan ^{-1}\left(\frac{2 \mu_{11}}{\mu_{20}-\mu_{02}}\right) .
\end{gathered}
$$




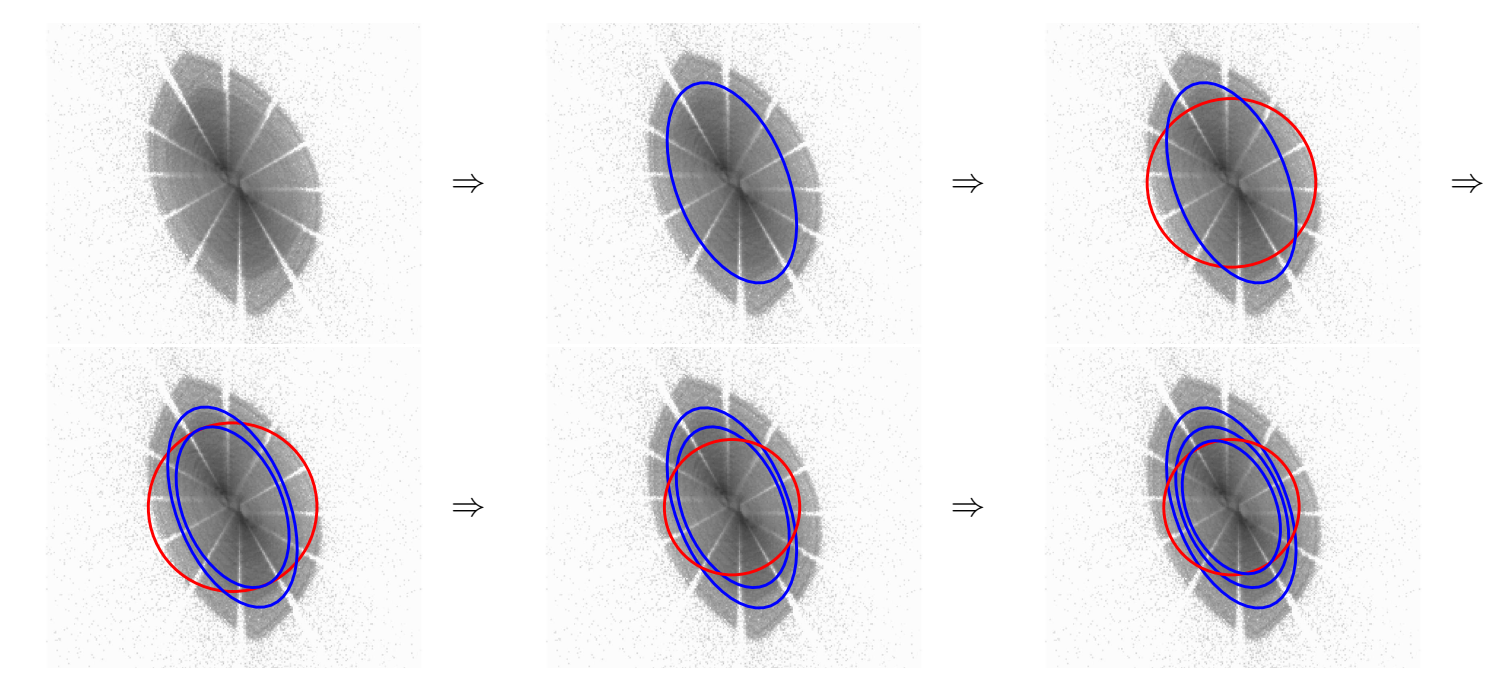

Figure 2. Ellipse fitting process. A sequence of elliptical fits and circular exclusion regions, illustrating the ellipse-fitting process. The first frame is the raw image. The second frame superimposes the initial ellipse. The third displays the exclusion circle, and the fourth, fifth, and sixth frames show the process repeated for the next two ellipses.

where $\bar{x}$ and $\bar{y}$ define the ellipse's center, $a$ and $b$ are the ellipse's semi-major and semi-minor axes, respectively, and $\phi$ is the position angle of the ellipse.

An initial ellipse was generated using this technique from the image. Subsequent ellipses were made by calculating the moments of a subimage containing only the pixels inside a circle of radius $0.8 a$ around the previous ellipse's center, and generating new ellipse parameters from those moments. Figure 2 illustrates a sequence of iterations of this process, with the fitted ellipses displayed alongside their 0.8 a "exclusion circles". This process was repeated until either an ellipse axis was smaller than one pixel, the parameters of a new ellipse was identical to the previous one, or a predetermined maximum number of fitted ellipses were produced. Generally, fitting was stopped by an ellipse axis growing smaller than one pixel.

The ECF for an ellipse was calculated by determining the fraction of counts enclosed by the ellipse:

$$
E C F=\frac{C_{\text {ellipse }}}{C_{\text {tot }}}
$$

where $C_{\text {ellipse }}$ is the number of counts inside an ellipse and $C_{\text {tot }}$ is the number of counts incident on the detector.

This technique sometimes produces ellipses that intersect previous ones. We do not want to include these because we are aiming for a simple, easy-to-use, and non-physical parameterization of the PSF; therefore, such ellipses were discarded. This ensures that smaller ellipses are completely enclosed within larger ones, which guarantees a monotonically increasing ECF as the semi-major axis increases. The exclusion circle for the next ellipse was based on the discarded ellipse to ensure that the unwanted ellipse would not be regenerated.

A set of ellipses with particular ECFs from each simulation is needed to facilitate useful comparisons. The ECFs of the ellipses generated by the moments do not fall into any pattern helpful for comparing to other simulations or observations, so we created a group of ellipses with ECFs that are multiples of $5 \%$ (e.g., $5 \%$, $10 \%, \ldots, 95 \%)$. We made these ellipses by interpolating between the two existing ellipses that bracketed the desired ECF (e.g., a ECF $=15 \%$ ellipse could have been produced by interpolating between an ECF $=12.32 \%$ ellipse and an ECF $=19.63 \%$ ellipse, if those were the ellipses with the closest ECF above and below 15\%). If the ECF of the original (i.e. largest) moment-derived ellipse was less than $95 \%$, the particular ellipses we want with ECFs greater than that ellipse's ECF were extrapolated from the original ellipse, freezing the position angle and ellipticity. 


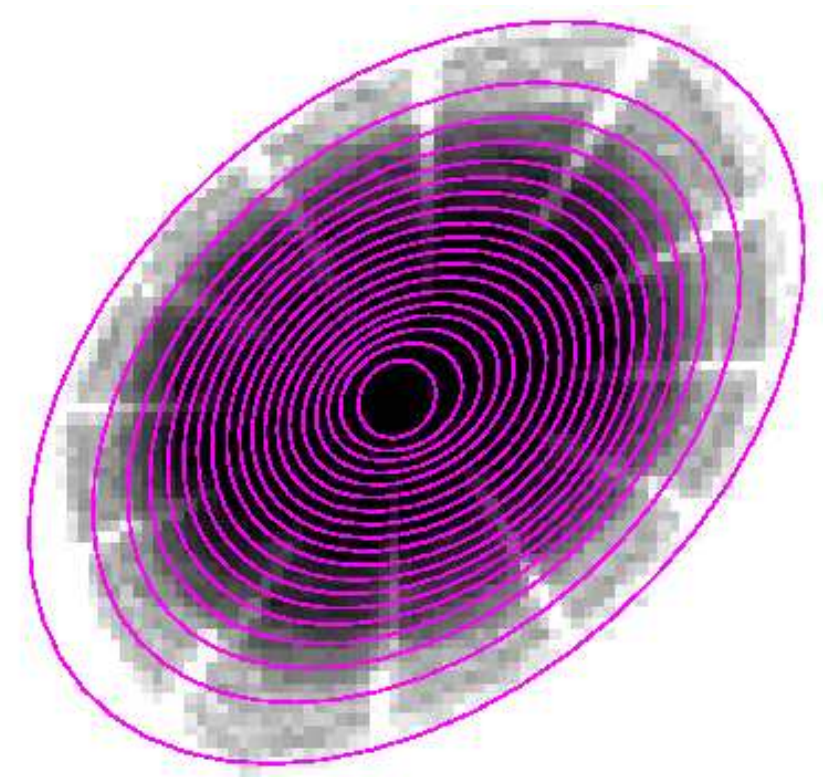

Figure 3. An image of the $0.277 \mathrm{keV}$ PSF at $10^{\prime}$ off-axis and $150^{\circ}$ azimuth overlaid with the ellipses for that pointing that have ECFs that are multiples of $5 \%$. The third-largest ellipse is the $85 \%$ ECF ellipse. Note that the innermost ellipse is offset, illustrating the difficulty in fitting the PSF's core.

Because the only way to measure the ECF is by summing within the ellipse, the initial interpolation (or extrapolation) almost never results in an ECF of exactly what was wanted. To get the particular value we want, we iterated until an ellipse with an ECF within 0.01\% of the desired, multiple-of-5\%, ECF was obtained. Figure 3 shows an example of this particular ellipse set for one PSF.

ECFs were measured by extracting the counts in each of the ellipses using the funcnts tool, a FUNTOOLS ${ }^{5}$ tool for summing the counts in a region of an image. Funcnts's summing algorithm includes all of a pixel or none; no partial pixel values are included. Figure 4 presents a selection of plots showing the ECF as a function of semi-major axis for the fitted ellipses. Figure 5 shows a selection of the fitted ellipses' semi-major axis vs off-axis angle.

\section{COMPARISON TO CHANDRA OBSERVATIONS}

Archival Chandra HRC-I observations of point sources at various pointings were compared with the simulations. Simulated PSFs exactly matching the sources' pointings were produced in order to get the best-case comparison between the raytrace ECF results and real Chandra data.

Observations were de-rolled, background subtracted, and the counts inside each simulation's ellipses were measured. The placement of the ellipses on the observational data was made by using a sigma-clipping centroiding algorithm to determine the center of both the observational data and the simulation. The offset of each ellipse from the simulation's centroid was calculated, and this offset was used to place the ellipse at the same relative position on the observational data.

The observation and simulation results were normalized by the background-subtracted counts inside the region defined by the simulation's ECF $=85 \%$ ellipse. This ellipse was chosen because it is large enough to include most of the PSF, but small enough to avoid the PSF wings (the correctness of the simulation in the PSF wings is uncertain), and to reduce the background-subtraction uncertainty (which would increase at larger ECFs). A selection of the ellipses with $85 \%$ ECFs are presented in Table 4.

The simulations and the observations were generally in agreement, within Poisson uncertainties, below the $85 \%$ normalization baseline. The Poisson uncertainties were usually smaller than $5 \%$, except in the core of PSFs at large off-axis angles, because of low counts in the observations in that region. See Figure 6 for examples. 
$O^{\prime}$ offaxis, $105^{\circ}$ azimuth

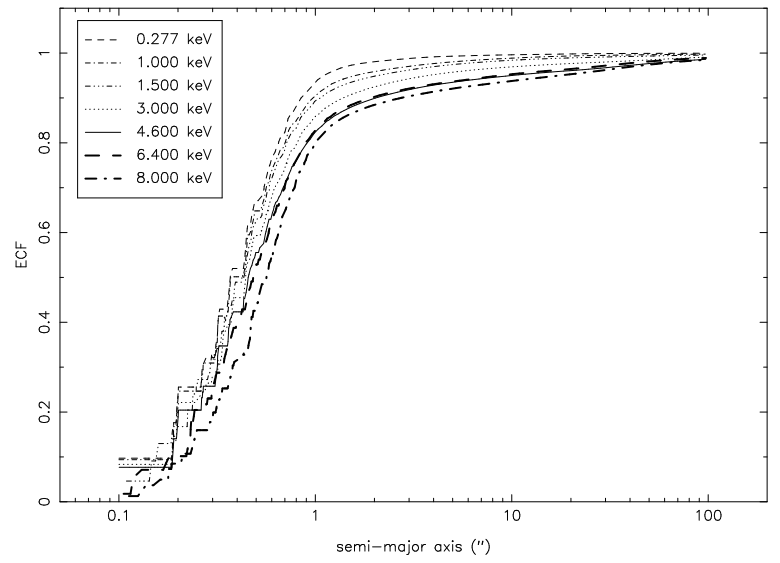

$10^{\prime}$ offaxis, $60^{\circ}$ azimuth

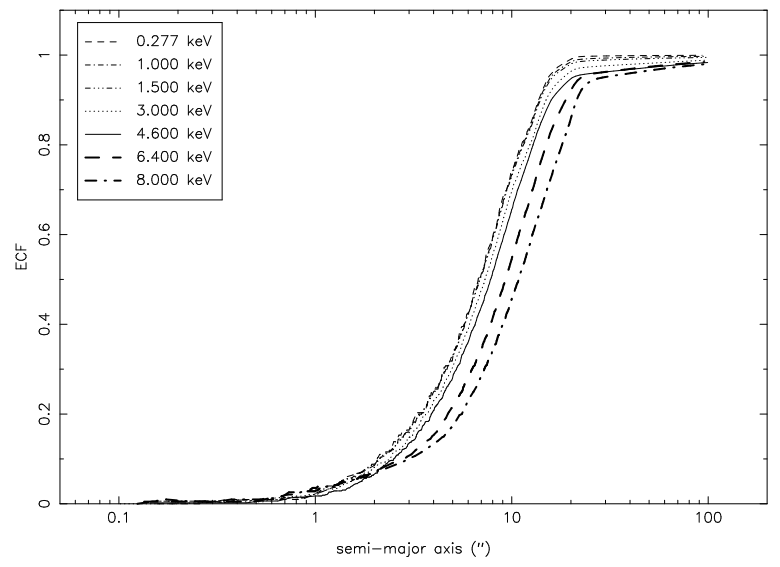

$5^{\prime}$ offaxis, $60^{\circ}$ azimuth

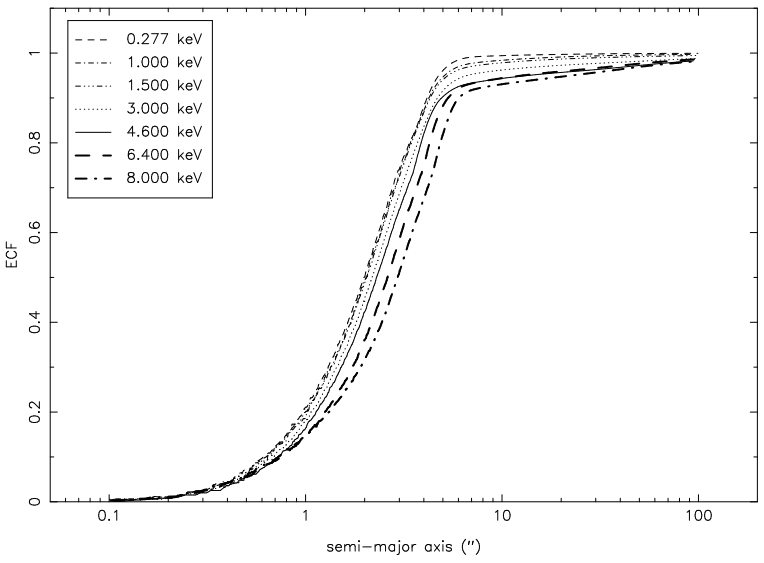

$15^{\prime}$ offaxis, $60^{\circ}$ azimuth

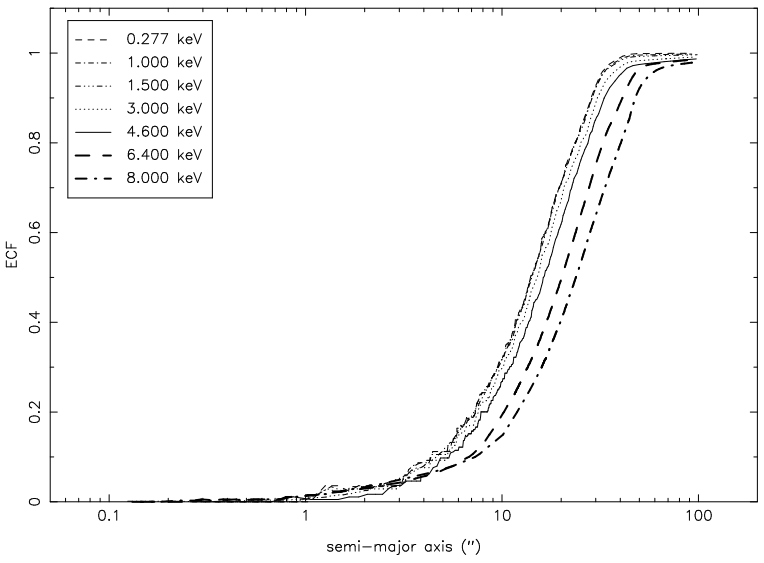

Figure 4. ECF as a function of semi-major axis for $0^{\prime}$ to $15^{\prime}$ off-axis and $60^{\circ}$ azimuth (the on-axis plot's azimuth angle is irrelevant). The jaggedness of the on-axis plot is caused by the relatively large size of the pixels in $\left(1 / 8^{\prime \prime}\right.$ in this case) relation to the overall (small) size of the on-axis PSF. Notice the increase in size of the PSF with both off-axis angle and energy. 


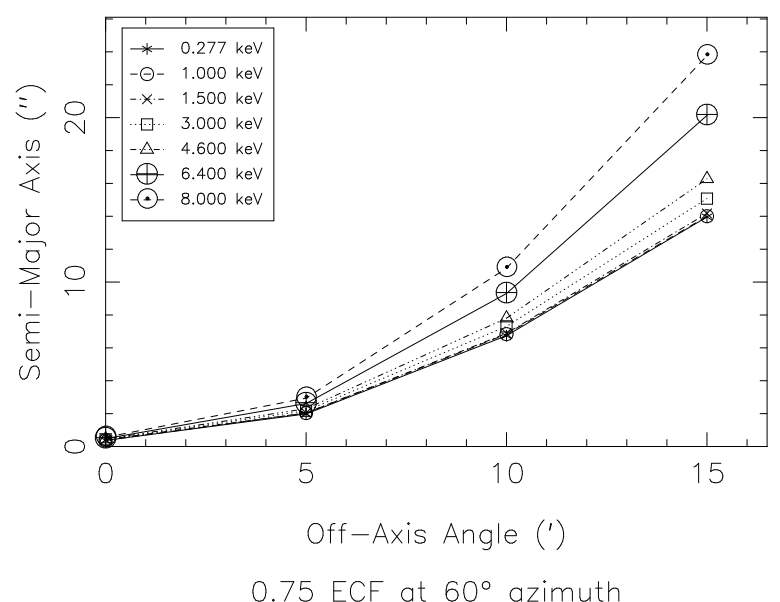

0.75 ECF at $60^{\circ}$ azimuth

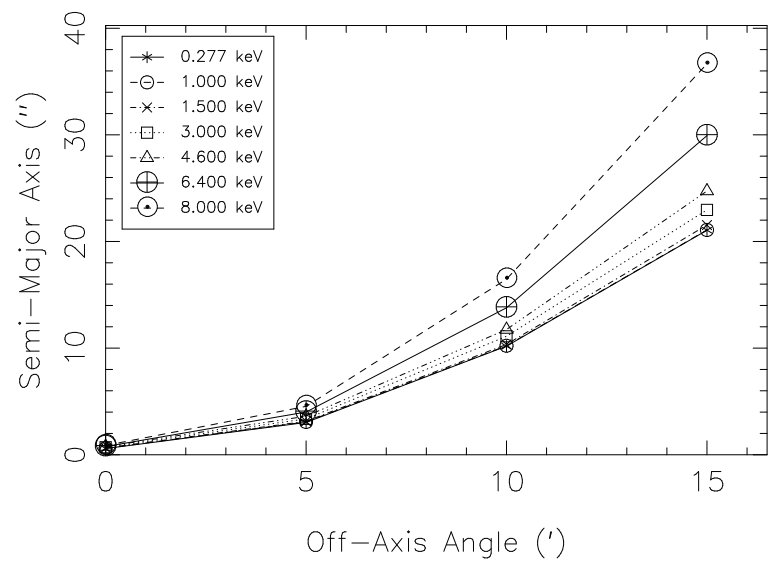

0.95 ECF at $60^{\circ}$ azimuth

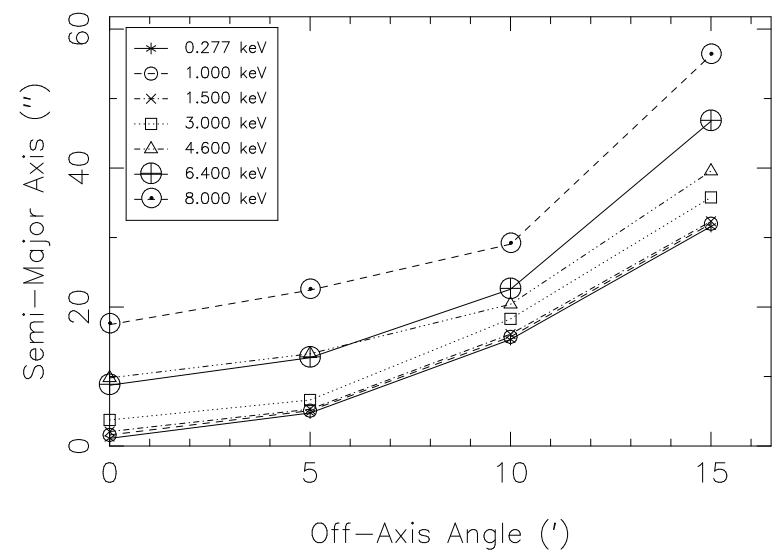

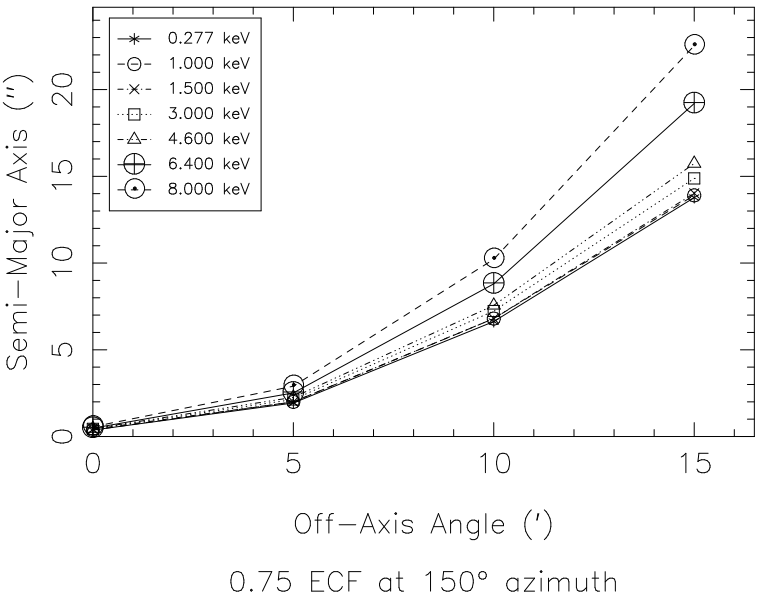

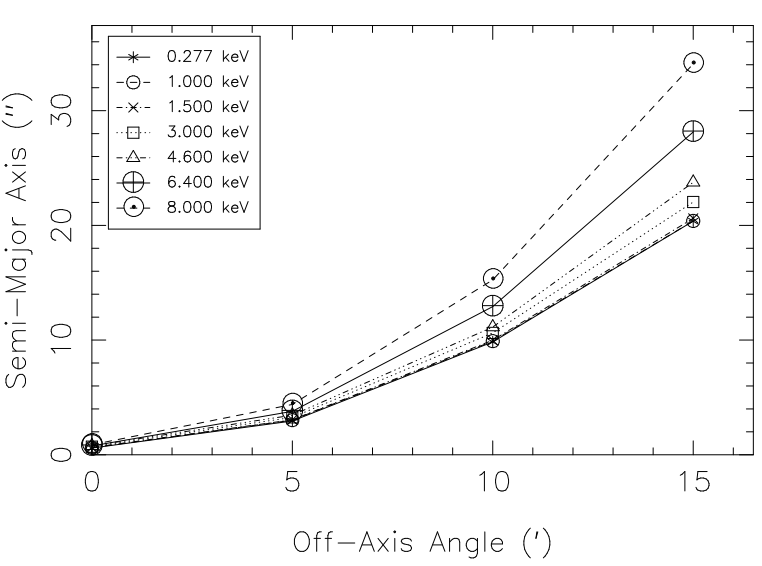

0.95 ECF at $150^{\circ}$ azimuth

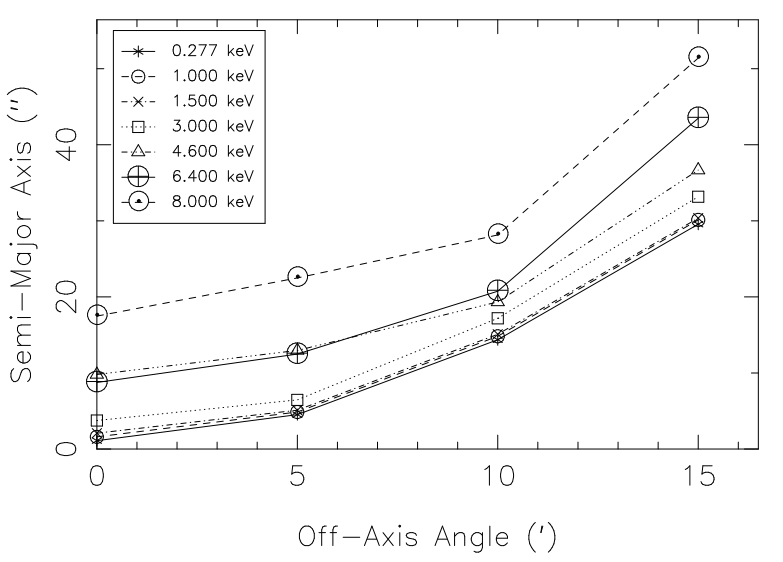

Figure 5. The semi-major axis of the ellipses with $\mathrm{ECF}=0.5,0.75$, and 0.95 as a function of off-axis angle, for $60^{\circ}($ left column) and $150^{\circ}$ (right column) azimuthal pointings. 


\begin{tabular}{|c|c|c|c|c|}
\hline Energy & $\begin{array}{c}\text { Off-axis } \\
\text { angle }\end{array}$ & $\begin{array}{c}\text { semi-major } \\
\text { axis }\end{array}$ & ellipticity & $\begin{array}{c}\text { position } \\
\text { angle }\end{array}$ \\
\hline \hline 0.277 & 0 & 0.72 & 0.03 & 5.67 \\
\hline 0.277 & 5 & 3.65 & 0.40 & -4.81 \\
\hline 0.277 & 10 & 12.16 & 0.32 & 1.34 \\
\hline 0.277 & 15 & 25.38 & 0.31 & 5.88 \\
\hline \hline 1.000 & 0 & 0.80 & 0.03 & 18.96 \\
\hline 1.000 & 5 & 3.76 & 0.39 & -4.22 \\
\hline 1.000 & 10 & 12.36 & 0.32 & 1.41 \\
\hline 1.000 & 15 & 25.55 & 0.31 & 5.97 \\
\hline \hline 1.500 & 0 & 0.83 & 0.02 & 23.12 \\
\hline 1.500 & 5 & 3.80 & 0.39 & -4.21 \\
\hline 1.500 & 10 & 12.45 & 0.32 & 1.44 \\
\hline 1.500 & 15 & 25.70 & 0.31 & 5.89 \\
\hline \hline 3.000 & 0 & 0.96 & 0.02 & 25.52 \\
\hline 3.000 & 5 & 4.07 & 0.38 & -3.34 \\
\hline 3.000 & 10 & 13.19 & 0.32 & 2.09 \\
\hline 3.000 & 15 & 27.40 & 0.32 & 6.72 \\
\hline \hline 4.600 & 0 & 1.16 & 0.01 & 33.48 \\
\hline 4.600 & 5 & 4.18 & 0.32 & -3.40 \\
\hline 4.600 & 10 & 13.86 & 0.32 & 3.20 \\
\hline 4.600 & 15 & 29.39 & 0.35 & 7.90 \\
\hline \hline 6.400 & 0 & 1.13 & 0.02 & 25.89 \\
\hline 6.400 & 5 & 4.57 & 0.35 & -1.08 \\
\hline 6.400 & 10 & 16.24 & 0.40 & 6.39 \\
\hline 6.400 & 15 & 35.92 & 0.48 & 10.16 \\
\hline \hline 8.000 & 0 & 1.31 & 0.04 & 29.54 \\
\hline 8.000 & 5 & 5.23 & 0.36 & -0.26 \\
\hline 8.000 & 10 & 19.37 & 0.46 & 7.68 \\
\hline 8.000 & 15 & 43.45 & 0.59 & 11.22 \\
\hline & & & & \\
\hline
\end{tabular}

Table 1. Parameters for ellipses with an ECF of $85 \%$ and an azimuth of $105 \%$. The semi-major axis and ellipticity generally increase with both energy and off-axis angle.

Several observations were in significant disagreement with the simulation ECF results above the $85 \%$ normalization point, with a constant offset between the observation and the simulation. See Figure 7 for examples of this offset. This seems to indicate that there is a systematic misestimation of the PSF in the simulation. More work is needed to better understand this discrepancy.

\section{CONCLUSIONS, DISCUSSION, AND FUTURE WORK}

This is a stable technique that produces usable results for parameterizing the size and shape of point sources at large off-axis angles. The systematic discrepancy between observations and our simulations shown in Figure 7 indicates that more work is needed to understand the properties of the simulated PSF.

It should be noted again that in the central core of the PSF, our ellipses tend to follow one of the central blob features. The PSF in the core is simply too complex to model with one ellipse. For subtraction or energy extraction from off-axis Chandra sources, this is not a concern, since such an analysis will be concerned with the entire PSF, not the fine structure of the core region. 


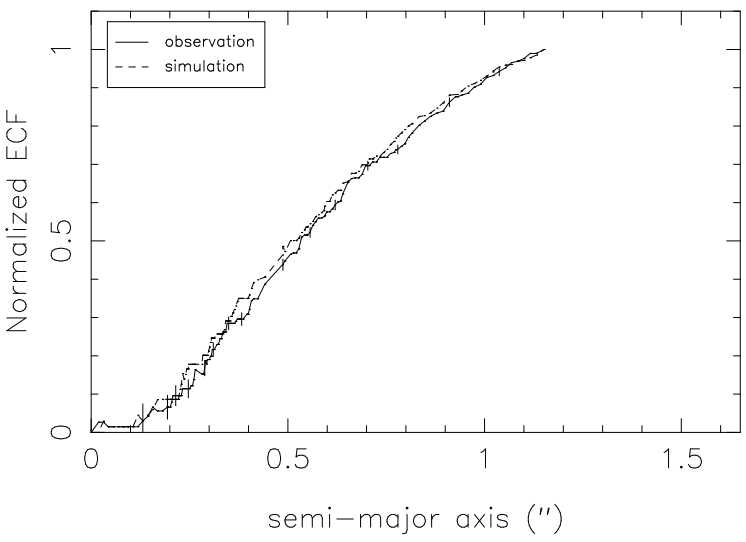

$10.006^{\prime}$ offaxis $271.32^{\circ}$ azimuth $0.5^{\prime \prime} /$ pixel

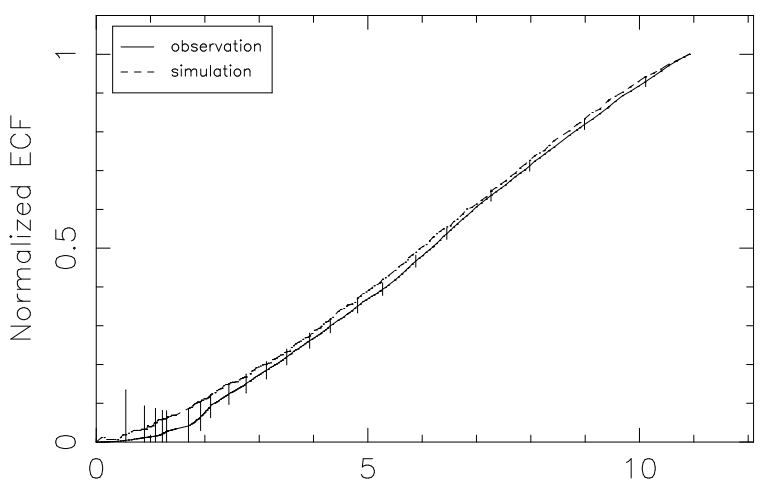

semi-major axis (")

$15.276^{\prime}$ offaxis $359.87^{\circ}$ azimuth $0.5^{\prime \prime} /$ pixel

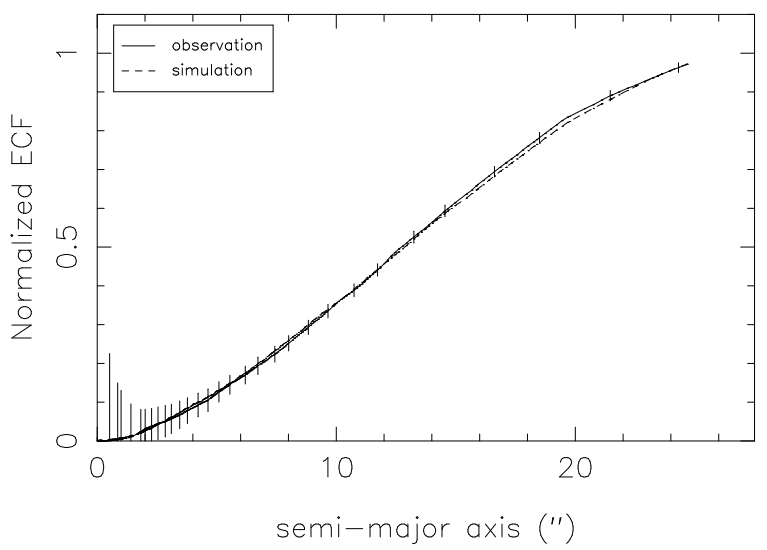

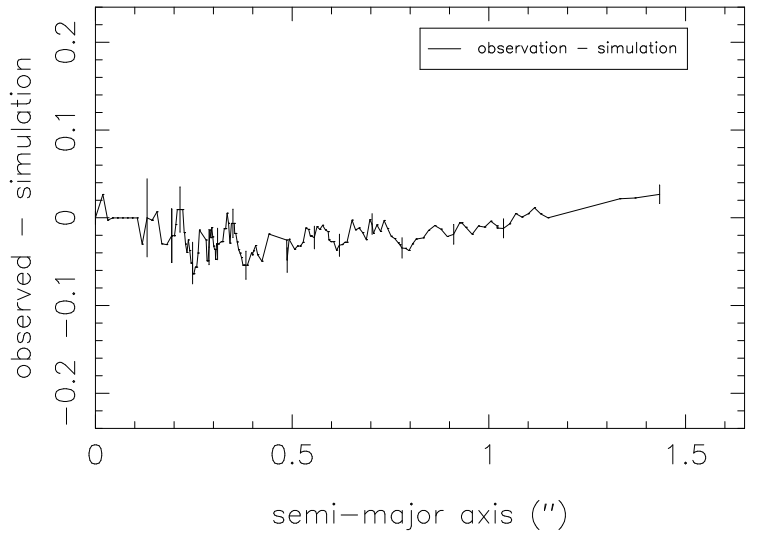

$10.006^{\prime}$ offaxis $271.32^{\circ}$ azimuth $0.5^{\prime \prime} /$ pixel
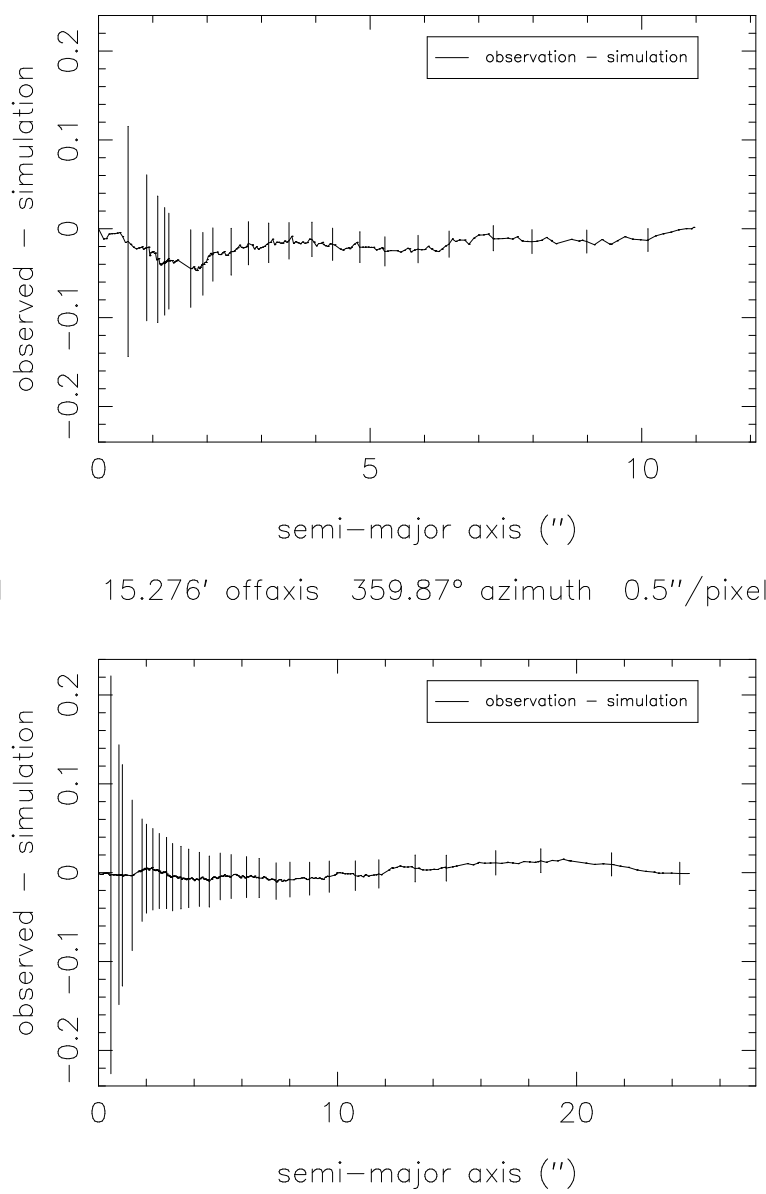

Figure 6. Left column: normalized ECF as a function of ellipse semi-major axis for three archival observations. Right column: residuals from the left column. Note that in the $4.468^{\prime}$ and $2.019^{\prime}$ observations, observation's normalized ECF is systematically offset by close to $5 \%$. 

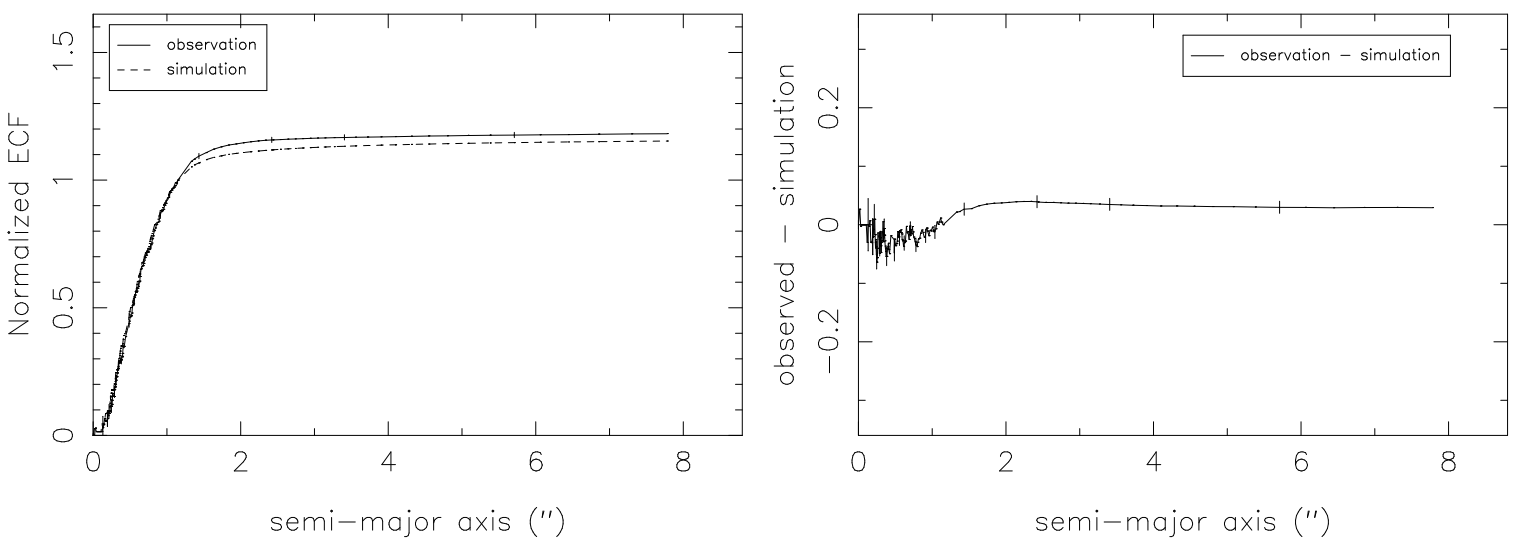

$10.006^{\prime}$ offaxis $271.32^{\circ}$ azimuth $0.5^{\prime \prime} /$ pixel

$10.006^{\prime}$ offaxis $271.32^{\circ}$ azimuth $0.5^{\prime \prime} /$ pixel
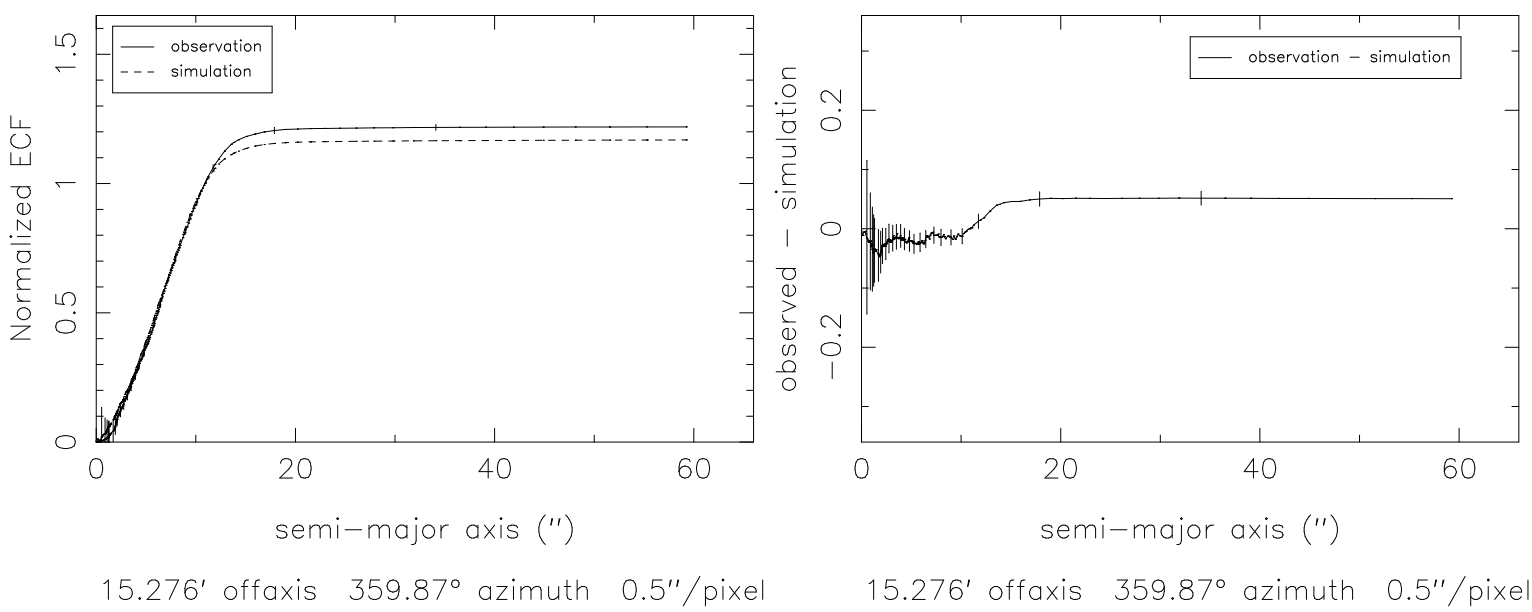

$15.276^{\prime}$ offaxis $359.87^{\circ}$ azimuth $0.5^{\prime \prime} /$ pixel

$5.276^{\prime}$ offaxis $359.87^{\circ}$ azimuth $0.5^{\prime \prime} /$ pixel
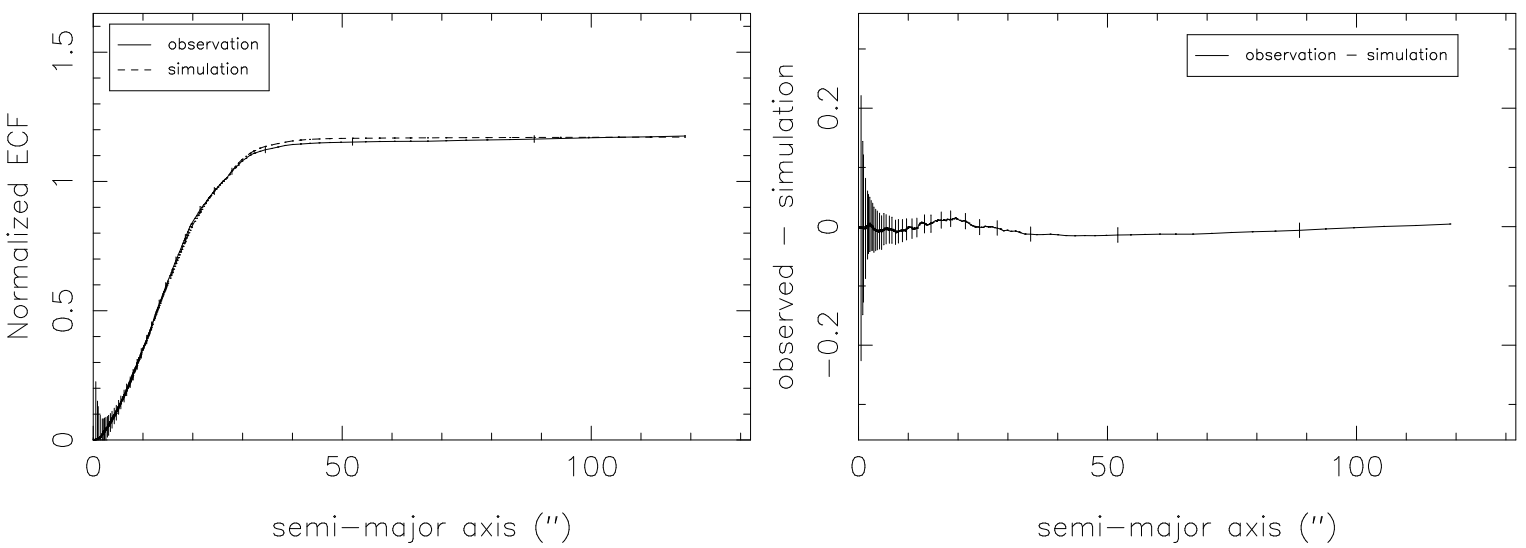

Figure 7. Left column: normalized ECF as a function of ellipse semi-major axis for three archival observations. Right column: residuals from the left column. Note that in the $4.468^{\prime}$ and $2.019^{\prime}$ observations, observation's normalized ECF is systematically offset by close to $5 \%$ at large semi-major axis values. 
To increase the precision of our final products, the ellipses will be generated and counts will be extracted directly from the simulated rays instead of being first binned into images.

\section{ACKNOWLEDGMENTS}

This work was supported by NASA contract NAS8-39073.

The authors would like to thank Richard J. Edgar and Leon van Speybroeck for their contributions.

\section{REFERENCES}

1. D. Jerius, L. Cohen, R. J. Edgar, et al., "The role of modeling in the calibration of Chandra's optics", in Proc. SPIE 5165, X-Ray and Gamma-Ray Instrumentation for Astronomy XIII, 2004, this volume.

2. The Chandra Proposer's Observatory Guide, Chapter 4. 5 ed. Dec. 2002 http://cxc.harvard.edu/proposer/POG/html/HRMA.html

3. The Chandra Proposer's Observatory Guide, Chapter 7. 5 ed. Dec. 2002 http://cxc.harvard.edu/proposer/POG/html/HRC.html

4. M. R. Teague, "Image Analysis via the General Theory of Moments", J. Opt. Soc. Am. 70, pp. 920-930, 1980 .

5. E. Mandel, S. S. Murray, and J. B. Roll, "Funtools: An Experiment with Minimal Buy-in Software", in ASP Conf. Ser., Vol. 238, Astronomical Data Analysis Software and Systems X", 225, 2001. 